\title{
Enabling Multi-Carrier Relay Selection by Sensing Fusion and Cascaded ANN for Intelligent Vehicular Communications
}

\author{
Shuping Dang, Member, IEEE, Miaowen Wen, Senior Member, IEEE, Shahid Mumtaz, Senior Member, \\ IEEE, Jun Li, Member, IEEE, and Chengzhong Li
}

\begin{abstract}
Cooperative relaying has been adopted as one of the most important techniques to enhance the energy efficiency and coverage. Multi-carrier relay selection is an efficient method to allocate spatial/spectral resources in cooperative relay networks and provides diversity gain. However, the implementation of multicarrier relay selection is not straightforward, and could render the high system complexity (for centralized implementation schemes) or long processing delay (for distributed implementation schemes). These issues hinder the promotion and implementation of multicarrier relay selection for intelligent vehicular communications. To mitigate aforementioned issues, we propose an enabling technique of multi-carrier relay selection based on sensing fusion (SF) and

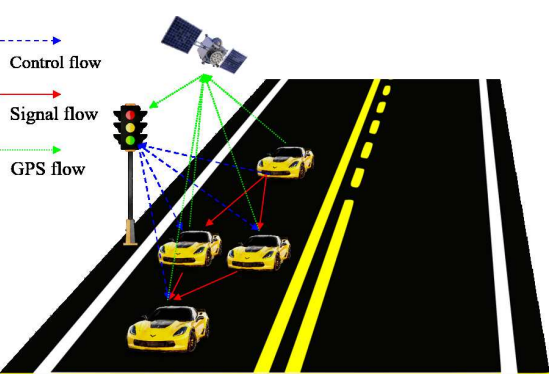
cascaded artificial neural networks (CANNs) for intelligent vehicular communications. We employ two well-known multicarrier relay selection schemes, i.e. bulk and per-subcarrier relay selection, to verify the effectiveness of the CANN based enabling technique. With the powerful processing ability with intelligent vehicles, the numerical results illustrate a promising vision of applying CANNs to enable multi-carrier relay selection for fast deployment in intelligent vehicular communication networks.
\end{abstract}

Index Terms-Artificial neural network (ANN), cooperative relaying, multi-carrier relay selection, sensing fusion (SF), vehicular communications.

\section{INTRODUCTION}

$\mathbf{I}$ $\mathrm{N}$ recent decades, traditional point-to-point communications that directly links the source and destination is unable to satisfy a variety of service requirements and is only applicable to some scenarios of near-field transmission [1]. To fulfill the increasingly high requirements of data rate, energy efficiency, coverage and reliability, cooperative relaying and relay-

This work was supported in part by Guangxi Natural Science Foundation under Grant AD19245043, in part by Nanning Excellent Young Scientist Program under Grant RC20190201, in part by Guangxi Beibu Gulf Economic Zone Major Talent Program, in part by the Pearl River Nova Program of Guangzhou under Grant 201806010171, in part by the Natural Science Foundation of Guangdong Province under Grant 2018B030306005

S. Dang is with the R\&D Center, Guangxi Huanan Communication Co., Ltd., Nanning 530007, China and is currently with Computer, Electrical and Mathematical Science and Engineering Division, King Abdullah University of Science and Technology (KAUST), Thuwal 239556900, Saudi Arabia (e-mail: shuping.dang@kaust.edu.sa).

$M$. Wen is with the School of Electronic and Information Engineering, South China University of Technology, Guangzhou 510641, China (email: eemwwen@scut.edu.cn).

S. Mumtaz is with Instituto de Telecomunicações, Aveiro 3810-193, Portugal (e-mail: smumtaz@av.it.pt).

$\mathrm{J}$. Li is with the School of Mechanical and Electrical Engineering, Guangzhou University, Guangzhou 510006, China (e-mail: lijun52018@gzhu.edu.cn).

C. $\mathrm{Li}$ is with the R\&D Center, Guangxi Huanan Communication Co., Ltd., Nanning 530007, China (e-mail: chengzhong.li@hncom.ac.cn). assisted communications have attracted many researchers' attention [2]. In order to further enhance the spectral efficiency of cooperative relaying and utilize the existing infrastructures in fourth generation (4G) wireless communication networks, multi-carrier transmission has been incorporated in cooperative relaying, which results in multi-carrier cooperative systems [3], [4]. This conception is believed to play a key role in even sixth generation (6G) networks [5].

For multi-carrier cooperative systems assisted by multiple relays, the most efficient technique to coordinate spatial and spectral resources and provide diversity gain refers to the multi-carrier relay selection. By multi-carrier relay selection, a two-dimensional space-frequency grid is formed and relays are selected based on this grid so as to optimize certain performance metrics [6]. In this way, transmitted signals on certain subcarriers will only be received and processed by optimized relays, so as to achieve a special spatial diversity gain termed the selection diversity gain. The primary work proposing multi-carrier relay selection was presented in 2007 [7], in which two basic selection criteria are given, i.e. bulk relay selection and per-subcarrier relay selection.

Following the pioneering work, more detailed analysis of multi-carrier relay selection is presented in [8]. In this work, uncoded decode-and-forward (DF) relay systems performing multi-carrier relay selection with local channel state infor- 
mation (CSI) is considered, and six selection metrics are analyzed. The above mentioned works only focus on multicarrier DF relaying. The first work dedicating to multi-carrier amplify-and-forward (AF) relaying is presented in [9], and several bounds of outage probabilities for multi-carrier AF relaying systems are consequently derived. The analytical methodology used in [9] is then extended to frequency correlated fading channels (i.e. the fading of each subcarrier is not independent) in [10]. In a similar manner, the bounds on the pairwise error probability (PEP) of multi-carrier AF relay systems with multi-carrier relay selection are calculated in [11]. To improve the tightness of the bound, the power series expansion and residue theorem are employed to yield another closed-form approximation of outage probability for both DF and AF relay systems with multi-carrier relay selection [12]. The first work analyzing multi-carrier relay selection in spatially correlated networks is given in [13]. Multi-carrier relay selection is incorporated with super dense networks, deviceto-device (D2D) communications, and index modulation (IM) in [14]-[18]. Based on these research works, a comprehensive theoretical framework of multi-carrier relay selection has been constructed.

It has been widely acknowledged that multi-carrier relay selection plays an irreplaceable role for performance enhancement. However, because relay nodes are spatially distributed in practice, coordination among multiple relays and implementation of a certain relay selection criterion in the multicarrier context are not trivial tasks [19]. Traditionally, multicarrier relay selection can be performed by either a centralized scheme or a decentralized scheme [20]. By the centralized scheme, a centralized and powerful controller is assumed to make decisions and announce the selected relays for all subcarriers via feedforward channels. Such a controller can exist at a base station (BS) or an access point (AP). However, it is required that the centralized controller performs complicated computation and would result in high system complexity. By the decentralized scheme, a timer-based implementation is applied, which does not require the collection of global CSI at a certain node, but leads to a higher processing delay. Albeit effective, its feasibility in practice, especially in vehicular communication networks where the end-to-end latency is critical, is still questionable.

Since the arrival of the 'smart era' of vehicular communications where most of vehicles have powerful processing capability and intelligent functionality, this provides a basis to enable multi-carrier relay selection in a more efficient manner based on artificial intelligence (AI) related technologies [21] [23]. Furthermore, due to the state-of-the-art achievements in channel estimations, channel statistics and relevant estimated states utilized for network optimization are normally labeled data from multiple sources [24]. For example, the channel state can can be defined by the link reliability measured by local transceivers at vehicles and the road infrastructure (light poles, electric charging piles, and inductive pavements), as well as the physical locations of vehicles measured through Global Positioning System (GPS) signals via satellites [25]. This makes supervised machine learning (ML) that relies on labeled fused data more suited for implementing multi-carrier relay selection in some circumstances [26]-[30].

In light of this, we propose an enabling technique for multi-carrier relay selection based on sensing fusion (SF) and supervised ML through cascaded artificial neural networks (CANNs) in intelligent vehicular communication systems and employ both bulk and per-sucbarrier relay selection schemes as examples to demonstrate the application of the enabling technique. This enabling technology benefits from the current developments of smart infrastructure and cloud/fog computing for vehicular communications [31]-[33].

The rest of this paper is organized as follows. In Section II, we present the system model studied in this paper and introduce the fundamentals of supervised ML via CANNs. Based on these, we in particular discuss the CANN based multi-carrier relay selection enabling techniques for bulk and per-subcarrier relay selection schemes in Section III and Section IV, respectively. Numerical results are presented and discussed in Section V. Finally, we conclude the paper in Section VI.

\section{FUNDAMENTALS}

\section{A. System Model}

In this paper, we consider a classic multi-carrier multi-relay cooperative communication network, comprising a source, a destination, and $M$ relays operating in the half-duplex mode. All $M$ relays are spatially distributed within a clustered area and ready to participate in forwarding signals from the source to the destination. We can denote the set of all $M$ relays as $\mathcal{M}$, and obviously $|\mathcal{M}|=M$. There are $K$ subcarriers that are assumed to be independent for use to convey signals in parallel. The set of $K$ subcarriers is denoted as $\mathcal{K}$, and obviously we have $|\mathcal{K}|=K$. For a classic multi-carrier multi-relay cooperative communication network, we have the following common assumptions to facilitate performance analysis and optimization $^{1}$

- Instantaneous CSI is perfectly known at the source and destination for resource optimization and information retrieval purposes.

- Synchronization in both time and frequency domains is perfect among all communication nodes.

- Network topology is unchanged over the entire transmission frame and all $M$ relays are located within a cluster, which is relatively small compared to the distance between the source and destination.

- The direct transmission link between the source and destination is blocked because of some reasons, e.g., overlong distance and severe shadowing. Consequently, the end-toend transmission from the source to the destination must be forwarded by one or more relays.

- Bits and power are equally allocated over all transmitters and subcarriers with transmit power $P_{t}$.

- The channel fading through $M$ relays over $K$ subcarriers (i.e. an $M \times K$ orthogonal transmission grid) is independently and identically distributed (i.i.d.) without

\footnotetext{
${ }^{1}$ These assumptions are applicable for both stationary communication networks and urban vehicular communication networks, where vehicles move at low speeds.
} 
any interference and correlation. The fading model and relevant distributions are unchanged.

- Noise at the receivers of all communication nodes is i.i.d. additive white Gaussian noise (AWGN) with average noise power $N_{0}$.

Based on the above assumptions, we leverage SF to integrate CSI from multiple sources. For each subcarrier relayed over an arbitrary relay, there are four information sources required to be integrated, which include the characteristic coefficients of the large-scale fading and the small-scale fading $p_{i}(m)$ and $q_{i}(m, k)$ for the first and second hops, respectively. In practice, the characteristic coefficients of the large-scale fading is treated as statistical CSI, which is dependent on the relative locations of source, relay, and destination nodes, as well as terrain. The small-scale fading coefficients are time- and frequency-varying due to multi-path effects, e.g., reflection, scattering, and diffraction. In this regard, the SF process should be two-stage. In the first stage, $p_{i}(m)$ and $q_{i}(m, k)$ in the same hop are fused to be the single-hop channel power gain $G_{i}(m, k)=\left(p_{i}(m)\right)^{-\alpha} q_{i}(m, k)$, where $\alpha$ is called the path loss exponent [19]. In the second stage, the single-hop gains pertaining to the first and second hops are fused to be the equivalent end-to-end channel power gain $G(m, k)$. The equivalent end-to-end channel power gain $G(m, k)$ characterizes the end-to-end transmission reliability forwarded by the $m$ th relay over the $k$ th subcarrier, which can be explicitly written as [13]

$$
G(m, k)=\min \left\{G_{1}(m, k), G_{2}(m, k)\right\}
$$

and

$$
G(m, k)=\frac{G_{1}(m, k) G_{2}(m, k)}{G_{1}(m, k)+G_{2}(m, k)+1},
$$

for DF and AF relay aided networks, respectively. The equivalent end-to-end channel power gain $G(m, k)$ is one of the major references for multi-carrier relay selection in practice, which is adopted as the performance metric for both bulk and per-subcarrier relay selection schemes in this paper. In practice, this gain is a representative of fused data originated from multiple sources (e.g., local measurements via the transceivers mounted at vehicles and remote measurements through GPS signals via satellites) and should be synthesized via SF processing. To facilitate information processing, we can also organize the equivalent end-to-end channel power gain $G(m, k)$ in the matrix form as follows:

$$
\begin{aligned}
\mathbf{G} & =\left[\begin{array}{cccc}
G(1,1) & G(1,2) & \ldots & G(1, K) \\
G(2,1) & G(2,2) & \ldots & G(2, K) \\
\vdots & \vdots & \ddots & \vdots \\
G(M, 1) & G(M, 2) & \ldots & G(M, K)
\end{array}\right] \\
& =[\mathbf{g}(1), \mathbf{g}(2), \ldots, \mathbf{g}(K)] \\
& =[\mathbf{h}(1), \mathbf{h}(2), \ldots, \mathbf{h}(M)]^{T},
\end{aligned}
$$

where $\mathbf{g}(k)=[G(1, k), G(2, k), \ldots, G(M, k)]^{T}$ and $\mathbf{h}(m)=$ $[G(m, 1), G(m, 2), \ldots, G(m, K)]$.

\section{B. Multi-Carrier Relay Selection}

Many research works have found that employing all available relays for forwarding the signals from the source to the

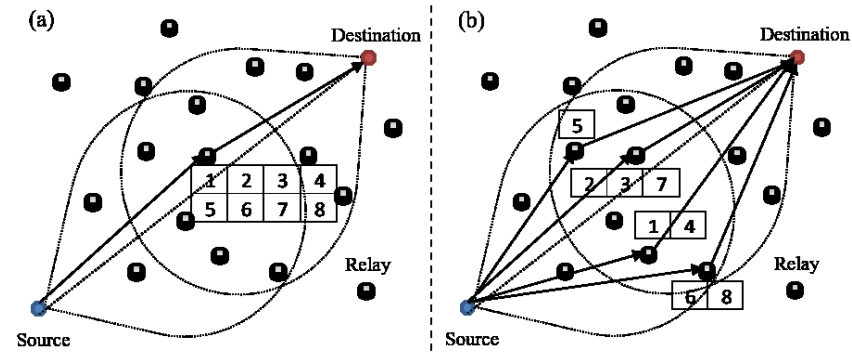

Fig. 1: Illustration of (a) bulk relay selection and (b) per-subcarrier relay selection with eight subcarriers, over which signals are transmitted in parallel. The boxes of numbers represent the indices of subcarriers.

destination is not the best strategy in practice [34]. This is because the exhaustive use of relays significantly degrades energy efficiency and could cause much higher signaling overhead for coordinating multiple relays. Moreover, the destination also needs advanced combining techniques to process replicas forwarded by multiple relays, which undoubtedly raises system complexity and latency. Further studies have also shown that selecting a single relay by a well-designed criterion can also produce full diversity for cooperative communication systems [35]. Therefore, relay selection has become a common resource optimization technique for cooperative communication systems, where there are multiple available relays for use. As we mentioned in the literature review in the introductory section, bulk relay selection and per-subcarrier relay selection are two basic relay selection schemes for multi-carrier multirelay cooperative systems. Both of them are capable of producing full diversity. The former only selects a single relay for forwarding all signals transmitted over all subcarriers, while the latter selects multiple relays in a per-subcarrier manner so that the optimal performance is achievable for signals transmitted over all subcarriers. We illustrate both selection schemes in Fig. 1 and expatiate on both multi-carrier relay selection schemes in the following paragraphs.

1) Bulk relay selection: According to the bulk relay selection criterion, only one relay in $\mathcal{M}$ is selected so that the worst channel in $\mathcal{K}$ can be enhanced. In this regard, the bulk relay selection criterion is given by

$$
\mathcal{M}_{\text {bulk }}=\{\tilde{m}\}=\bigcup_{k \in \mathcal{K}}\{\tilde{m}(k)\}=\arg \max _{m \in \mathcal{M}} \min _{k \in \mathcal{K}}\{G(m, k)\},
$$

where $\tilde{m}=\tilde{m}(1)=\tilde{m}(2)=\cdots=\tilde{m}(K)$ is the index of the selected relay in bulk for all $K$ subcarriers, and $\tilde{m}(k)$ denotes the index of the selected relay for the $k$ th subcarrier, $\forall k \in \mathcal{K}$. Obviously, because only one relay is selected, bulk relay selection is easy-to-implement without requirements of coordination and synchronization among relays. On the other hand, such a simplicity is obtained at the cost of lower reliability [20].

2) Per-subcarrier relay selection: Different from bulk relay selection, without the constraint on the number of selected relays, the per-subcarrier relay selection criterion allows selecting multiple relays up to $K$ so that each subcarrier can be processed and forwarded by the optimal relay. Relaying in 
such a per-subcarrier manner results in a higher throughput and better reliability than that of bulk relay selection. The per-subcarrier relay selection criterion can be written as

$$
\mathcal{M}_{p s}=\bigcup_{k \in \mathcal{K}}\{\tilde{m}(k)\}=\bigcup_{k \in \mathcal{K}}\left\{\arg \max _{m \in \mathcal{M}}\{G(m, k)\}\right\} .
$$

From the above criterion, it is evident that much higher system complexity and signaling overhead are raised by performing per-subcarrier relay selection, because multiple relays need to be coordinated in order to successfully implement the forwarding task.

\section{Supervised Machine Learning via Cascaded Artificial Neural Networks}

Supervised ML is one of the fundamental ML techniques and is effective for model-driven learning. In order to perform supervised ML, training data sets with labels are necessary. Because most communication nodes are expected to be equipped with channel estimation functionality in next generation networks, the CSI represented by $\{G(m, k)\}$ is assumed to be perfectly known. Also, by the relay selection criteria given in (4) and (5), we can also employ brute-force search algorithms to determine the optimal sets of relays $\mathcal{M}_{(\cdot)}$, i.e. labels, corresponding to given $\{G(m, k)\}$. With both training data sets and labels as pairs, the prerequisite for utilizing supervised ML is accomplished. Meanwhile, to implement the algorithms of supervised ML, we also need to construct an ANN with a back-propagation mechanism. A typical fully connected ANN consisting of $L$ layers, in which each layer has $N_{l}$ neurons, $\forall l \in[0, L]$, is illustrated in Fig. 2 .

In principle, given a training data set in a certain form as input, an ANN processes it by computing over neurons in multiple layers and produces the output in a certain form, which can be used to retrieve an estimated set of selected relay(s), say $\hat{\mathcal{M}}_{(\cdot)}$. This output will be compared with the expected output, i.e. the label, produced by brute-force search algorithms according to (4) and (5). Then, an error vector and an error gradient vector can be yielded to adjust the link weights and neuron activation thresholds layer by layer via the back-propagation mechanism. If a large group of training data sets with labels can be used to train the ANN in an iterative manner, the ANN is expected to provide an optimized set of selected relay(s) that is close to or even the same as the one produced by brute-force search algorithms. Therefore, the ANN can be trained off-line and then used on-line to process instantaneous CSI to quickly produce a multi-carrier relay selection strategy. The feasibility of using ANN to implement supervised ML relies on the unchanged statistical distribution of input data training sets. For an ANN with given architecture characterized by the network depth and the numbers of neurons, the training outcomes are a set of $\sum_{j=1}^{L-1} N_{j} N_{j+1}$ link weights connecting neurons in adjacent layers and a set of $\sum_{j=1}^{L} N_{j}$ activation thresholds pertaining to all neurons.

However, recent studies have confirmed that ANNs can function well for solving simple optimization questions, but are less capable of solving complex optimization questions, especially when the optimization dimension is higher than one [26]. The estimated solution produced by ANNs will never converge to the optimal one regardless of how many training episodes are involved. Unfortunately, this incapability might not be overcome by increasing the ANN depth or the number of neurons in each layer [23]. As a result, for solving multicarrier relay selection problems that are of two dimensions of space and frequency, conventional ANNs are of limited usefulness.

To enable multi-carrier relay selection using supervised ML, we resort to the CANN. Specifically, contrary to implementing the multi-carrier relay selection criteria in only one attempt, we split the computation of multi-carrier relay selection into two steps. Subsequently, we can construct the CANN by primary $\mathrm{ANN}(\mathrm{s})$ and secondary $\mathrm{ANN}(\mathrm{s})$ that perform the first-step computation and second-step computation, respectively. The inputs of primary $\mathrm{ANN}(\mathrm{s})$ are reorganized from training data sets, and the outputs of primary ANN(s) are the inputs of the secondary ANN(s). The final optimization solution yielded by the CANN can be retrieved by the outputs of secondary ANN(s). By such a cascaded structure, the training efficiency of supervised ML is expected to be enhanced, especially when the optimization dimension is higher than one and/or the optimization problem is non-linear [36], [37].

\section{Bulk Relay Selection Enabled by Cascaded ARTificial Neural NetWorks}

Contrary to the classic ANN without the cascaded structure, there is not a universal CANN suited for most optimization problems, but the CANN needs to be designed for each specific optimization problem. The design guideline of CANN is the optimization model and objective. For the bulk and persubcarrier relay selection problems, the design guidelines of CANN are the relay selection criteria given in (4) and (5), respectively. In this section, we first detail the architecture design of the CANN for bulk relay selection and its implementation method.

\section{A. Architecture Design of Cascaded Artificial Neural Networks}

By observing (4), the computation of bulk relay selection can be split into two steps. In the first step, the minimum channel power gain over all $K$ subcarriers forwarded by the $m$ th relay is searched. In the second step, the maximum of the searched minima over all $M$ relays is searched, which yields the index of the selected relay $\tilde{m}$ corresponding to the maximum. In this regard, we can construct $M$ primary ANNs that function as producing the minimum channel power gains over all $K$ subcarriers forwarded by $M$ relays. Then, we can have a single secondary ANN taking these minimum channel power gains as input and outputting the index of the relay corresponding to the maximum of the secondary inputs. For bulk relay selection, we further stipulate that all $M$ primary ANNs for $M$ relays are structurally homogeneous for simplicity, which implies that all $M$ primary ANNs share the same network depth and the number of neurons in each layer. The technical specifications of the primary and secondary 


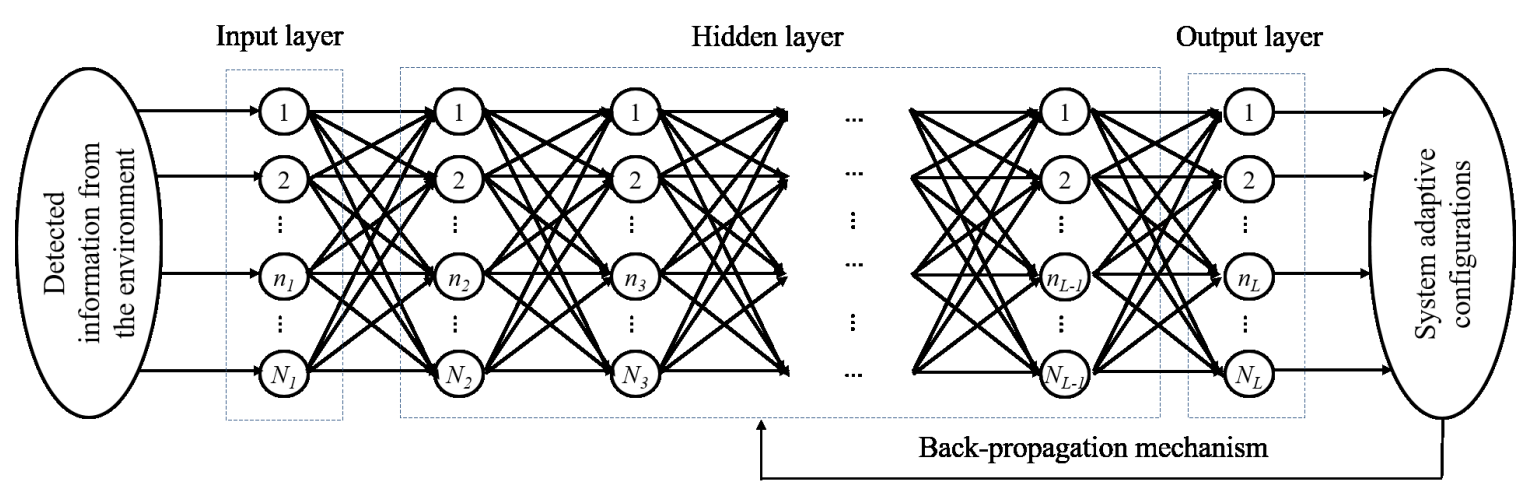

Fig. 2: Fully connected ANN model consisting of $L$ layers, among which there is a single input layer, a single output layer, and $L-2$ hidden layers.

TABLE I: Technical specification of the $m$ th primary ANN for performing bulk relay selection, $\forall m \in \mathcal{M}$.

\begin{tabular}{cl}
\hline Attribute & Explanation \\
\hline $\begin{array}{c}\text { Functiona- } \\
\text { lity }\end{array}$ & $\begin{array}{l}\text { Estimate the minimum of the input arguments: } \\
\hat{G}_{\min }(m) \approx G_{\min }(m)=\min _{k \in \mathcal{K}}\{G(m, k)\} .\end{array}$ \\
\hline \multirow{2}{*}{ Denotation } & $\begin{array}{l}\text { The number of layers is denoted as } L_{b u l k}^{p r}, \text { and the } \\
\text { number of neurons in the lth layer is denoted as } N_{b u l k}^{p r}(l) .\end{array}$ \\
\hline Input & $\begin{array}{l}\text { There are } K \text { inputs corresponding to }\{G(m, k)\}, \\
\forall k \in \mathcal{K}, \text { forwarded by the } m \text { th relay, i.e. } \mathbf{h}(m) .\end{array}$ \\
\hline \multirow{2}{*}{ Processing } & $\begin{array}{l}\text { Use the logistic function } f_{A}(x)=1 /(1+\exp (-x)) \text { as } \\
\text { the activation function and gradient descent algorithm to } \\
\text { enable back-propagation and dynamical link weight and } \\
\text { activation threshold adjustments with learning rate } \alpha_{b u l k}^{p r} .\end{array}$ \\
\hline Output & The estimated minimum of the input arguments $\hat{G}_{\text {min }}(m)$. \\
\hline $\begin{array}{c}\text { Initialization } \\
\text { method }\end{array}$ & $\begin{array}{l}\text { Random initialization for link weights and activation } \\
\text { thresholds with range expansion coefficient } \beta_{b u l k}^{p r} .\end{array}$ \\
\hline $\begin{array}{c}\text { Termination } \\
\text { condition }\end{array}$ & All labeled training data sets have been exhaustively used. \\
\hline
\end{tabular}

TABLE II: Technical specification of the secondary ANN for performing bulk relay selection.

\begin{tabular}{|c|c|}
\hline Attribute & Explanation \\
\hline $\begin{array}{c}\text { Functiona- } \\
\text { lity }\end{array}$ & $\begin{array}{l}\text { Estimate the index of the relay corresponding to the } \\
\text { maximum of the input arguments } \hat{G}_{\min }(m): \\
\hat{m} \approx \arg \max _{m \in \mathcal{M}}\left\{\hat{G}_{\min }(m)\right\} .\end{array}$ \\
\hline Denotation & $\begin{array}{l}\text { The number of layers is denoted as } L_{b u l k}^{s e} \text {, and the } \\
\text { number of neurons in the } l \text { th layer is denoted as } N_{b u l k}^{s e}(l) \text {. }\end{array}$ \\
\hline Input & $\begin{array}{l}\text { There are } M \text { inputs corresponding to }\left\{\hat{G}_{\min }(m)\right\} \text { from } \\
M \text { primary ANNs, } \forall m \in \mathcal{M} \text {. }\end{array}$ \\
\hline Processing & $\begin{array}{l}\text { Use the logistic function } f_{A}(x)=1 /(1+\exp (-x)) \text { as } \\
\text { the activation function and gradient descent algorithm to } \\
\text { enable back-propagation and dynamical link weight and } \\
\text { activation threshold adjustments with learning rate } \alpha_{\text {bulk }}^{\text {se }} \text {. }\end{array}$ \\
\hline Output & $\begin{array}{l}\text { The estimated vector } \hat{\mathbf{m}} \text {, in which the largest entry } \\
\text { indicates the index of the selected relay. }\end{array}$ \\
\hline $\begin{array}{c}\text { Initialization } \\
\text { method }\end{array}$ & $\begin{array}{l}\text { Random initialization for link weights and activation } \\
\text { thresholds with range expansion coefficient } \beta_{b u l k}^{s e}\end{array}$ \\
\hline $\begin{array}{l}\text { Termination } \\
\text { condition }\end{array}$ & All labeled training data sets have been exhaustively used. \\
\hline
\end{tabular}

ANNs for performing bulk relay selection are given in Table I and Table II. The CANN model for performing bulk relay selection is illustrated in Fig. 3.

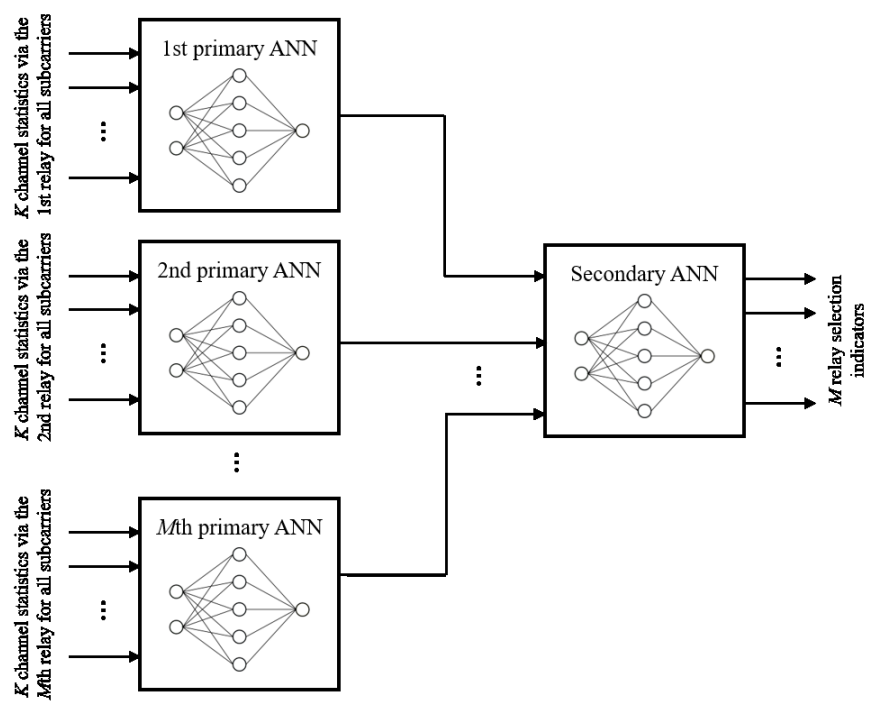

Fig. 3: CANN model for performing bulk relay selection with $M$ primary ANNs and a single secondary ANN.

\section{B. Implementation Method}

As $\{G(m, k)\}$ is normally exponentially or gamma distributed ranging from 0 to infinity [38], it is imperative to normalize the input data before passing them to CANNs in order to avoid ANN parameter saturation and enhance training efficiency. Here, we implement a simplistic and useful normalization mapping as

$$
G(m, k) \leftarrow \frac{G(m, k)-\min _{m \in \mathcal{M}, k \in \mathcal{K}}\{G(m, k)\}}{\max _{m \in \mathcal{M}, k \in \mathcal{K}}\{G(m, k)\}-\min _{m \in \mathcal{M}, k \in \mathcal{K}}\{G(m, k)\}},
$$

so that the input range can be restricted between 0 and 1 .

Then, with the normalized inputs, the initialization and training procedures of the primary and secondary ANNs can be carried out by the classic supervised ML supported by ANN as described in [22], given $L_{b u l k}^{p r},\left\{N_{b u l k}^{p r}(l)\right\}, \alpha_{b u l k}^{p r}, \beta_{b u l k}^{p r}$, $L_{b u l k}^{s e},\left\{N_{b u l k}^{s e}(l)\right\}, \alpha_{b u l k}^{s e}$, and $\beta_{\text {bulk }}^{\text {se }}$. The training procedures are operated in an iterative manner to update the link weights and activation thresholds so that the outputs given by ANNs can approach the desirable results, i.e. the labels. Such an iterative procedure will be terminated once all training data 
sets have been exhaustively used. Note that, in order to avoid overfitting, we do not allow repeated training by utilizing the same data sets. Details of the classic initialization and training procedure have been described in [22], which are not repeated here for saving space.

Meanwhile, because the entries of the output vector $\hat{\mathbf{m}}$ of the secondary ANN are normally decimals, they should be quantized for explicitly indicating which relay(s) should be selected. For bulk relay selection, by which there will be only one selected relay, we can simply define the quantized output vector $\hat{\mathbf{m}}$ as $\Xi_{b u l k}(\hat{\mathbf{m}})$, where $\Xi_{b u l k}(\cdot)$ is an entry-wise quantization function converting the largest entry to one and other entries to zeros.

To measure the training performance of the constructed CANN for performing bulk relay selection, we can employ the following performance evaluation metrics. First, we define the primary mean squared error (PMSE) as

$$
\begin{array}{r}
\text { PMSE }=\mathbb{E}\left\{\|\left[G_{\min }(1)-\hat{G}_{\min }(1), G_{\min }(2)-\hat{G}_{\min }(2),\right.\right. \\
\left.\left.\ldots, G_{\min }(M)-\hat{G}_{\min }(M)\right]^{T} \|_{F}^{2}\right\},
\end{array}
$$

to measure the training performance of the primary ANN, where $\mathbb{E}\{\cdot\}$ and $\|\cdot\|_{F}$ denote the expectation and Frobenius norm of the enclosed matrix/vector, respectively.

Similarly, we can define the secondary MSE (SMSE) as

$$
\mathrm{SMSE}=\mathbb{E}\left\{\|\mathbf{m}-\hat{\mathbf{m}}\|_{F}^{2}\right\},
$$

to measure the training performance of the secondary ANN, where $\mathbf{m}$ is an $M \times 1$ vector produced by brute-force search algorithms by (4) or (5), in which the entries corresponding to the selected relays are ones, while the entries corresponding to the other relays are zeros.

To measure the overall performance of the CANN by post-processing for bulk relay selection, we resort to the mean squared quantized error (MSQE) and relative error (RE) defined as

$$
\mathrm{MSQE}=\mathbb{E}\left\{\left\|\mathbf{m}-\Xi_{\text {bulk }}(\hat{\mathbf{m}})\right\|_{F}^{2}\right\},
$$

and

$$
\mathrm{RE}=\frac{\left|\min _{k \in \mathcal{K}}\{G(\tilde{m}(k), k)\}-\min _{k \in \mathcal{K}}\{G(\hat{m}(k), k)\}\right|}{\min _{k \in \mathcal{K}}\{G(\tilde{m}(k), k)\}},
$$

where $\hat{m}(k)$ can be extracted from $\mathbf{m}$, which is the index of the estimated optimal relay forwarding the signal transmitted over the $k$ th subcarrier.

\section{Per-Subcarrier Relay Selection Enabled by Cascaded Artificial Neural Networks}

Similar to the last section, in this section, we detail the architecture design of the CANN for per-subcarrier relay

\begin{tabular}{|c|c|}
\hline Attribute & Explanation \\
\hline $\begin{array}{l}\text { Functiona- } \\
\text { lity }\end{array}$ & $\begin{array}{l}\text { Estimate the index of the relay corresponding to the } \\
\text { maximum of the input arguments } G(m, k), \\
\forall m \in \mathcal{M}: \hat{m}(k) \approx \arg \max _{m \in \mathcal{M}}\{G(m, k)\} .\end{array}$ \\
\hline Denotation & $\begin{array}{l}\text { The number of layers is denoted as } L_{p s}^{p r} \text {, and the } \\
\text { number of neurons in the } l \text { th layer is denoted as } N_{p s}^{p r}(l) \text {. }\end{array}$ \\
\hline Input & $\begin{array}{l}\text { There are } M \text { inputs corresponding to }\{G(m, k)\}, \\
\forall m \in \mathcal{M} \text {, for the } k \text { th subcarrier i.e. } \mathbf{g}(k) \text {. }\end{array}$ \\
\hline Processing & $\begin{array}{l}\text { Use the logistic function } f_{A}(x)=1 /(1+\exp (-x)) \text { as } \\
\text { the activation function and gradient descent algorithm to } \\
\text { enable back-propagation and dynamical link weight and } \\
\text { activation threshold adjustments with learning rate } \alpha_{p s}^{p r} \text {. }\end{array}$ \\
\hline Output & $\begin{array}{l}\text { The vector } \hat{\mathbf{m}}(k) \text { containing } M \text { indicators estimating } \\
\text { which relay is selected for forwarding the } k \text { th subcarrier. }\end{array}$ \\
\hline $\begin{array}{l}\text { Initialization } \\
\text { method }\end{array}$ & $\begin{array}{l}\text { Random initialization for link weights and activation } \\
\text { thresholds with range expansion coefficient } \beta_{p s}^{p r} \text {. }\end{array}$ \\
\hline $\begin{array}{l}\text { Termination } \\
\text { condition }\end{array}$ & All labeled training data sets have been exhaustively used. \\
\hline
\end{tabular}
selection and its implementation method according to the selection criterion given in (5).
TABLE III: Technical specification of the $k$ th primary ANN for performing per-subcarrier relay selection, $\forall k \in \mathcal{K}$.

\section{A. Architecture Design of Cascaded Artificial Neural Networks}

Observing (5), we can find the most explicit difference between per-subcarrier relay selection and bulk relay selection to be the per-subcarrier property. That is, each subcarrier is treated independently in the selection procedure without considering other subcarriers, so that multiple relays could be selected for forwarding signals over multiple subcarriers. Based on this crucial observation, it is apparent that there needs to be $K$ primary ANNs corresponding to $K$ subcarriers. Each primary ANN has $M$ inputs related to the channel power gains of the $k$ th subcarrier forwarded by $M$ different relays and $M$ outputs indicating which input is the maximum (the corresponding output is expected to be one and the other outputs are expected to be zeros). In essence, these primary ANNs function as classifiers. Again, all these primary ANNs are homogeneous and share the same architecture. Subsequently, there are $M$ secondary ANNs taking the outputs from the primary ANNs as inputs. In particular, for the $m$ th secondary ANN, there are $K$ inputs that are the $m$ th outputs of all $K$ primary ANNs, and there is only one output that is binary, indicating whether the $m$ th relay should be selected or not. Each secondary ANN works individually as an OR gate with $K$ imperfect inputs, and all these $M$ secondary ANNs work on a distributed basis to function as a classifier. Likewise, they are also set to be homogeneous. The technical specifications of the primary and secondary ANNs are given in Table III and Table IV. The CANN model for performing per-subcarrier relay selection is shown in Fig. 4.

\section{B. Implementation Method}

To facilitate the processing of CANN and enhance the training efficiency, we also need to normalize $\{G(m, k)\}$ in exactly the same way as given in (6), which restricts the input ranging within 0 and 1 . Then, assigning $L_{p s}^{p r},\left\{N_{p s}^{p r}(l)\right\}$, $\alpha_{p s}^{p r}, \beta_{p s}^{p r}, L_{p s}^{s e},\left\{N_{p s}^{s e}(l)\right\}, \alpha_{p s}^{s e}$, and $\beta_{p s}^{s e}$ allows initializing and training the CANN for per-subcarrier relay selection by 
TABLE IV: Technical specification of the $m$ th secondary ANN for performing per-subcarrier relay selection, $\forall m \in \mathcal{M}$.

\begin{tabular}{cl}
\hline $\begin{array}{c}\text { Attribute } \\
\text { lity }\end{array}$ & Explanation \\
\hline Denotation & $\begin{array}{l}\text { The number of layers is denoted as } L_{p s}^{s e} \text {, and the } \\
\text { number of neurons in the } l \text { th layer is denoted as } N_{p s}^{s e}(l) .\end{array}$ \\
\hline Input & $\begin{array}{l}\text { There are } K \text { inputs that are the } m \text { th entries of } \hat{\mathbf{m}}(k), \\
\forall k \in \mathcal{K} .\end{array}$ \\
\hline Processing & $\begin{array}{l}\text { Use the logistic function } f_{A}(x)=1 /(1+\exp (-x)) \text { as } \\
\text { the activation function and gradient descent algorithm to } \\
\text { enable back-propagation and dynamical link weight and } \\
\text { activation threshold adjustments with learning rate } \alpha_{p s}^{s e} .\end{array}$ \\
\hline Output & $\begin{array}{l}\text { There is only a single output } \hat{m} \text { that is either } 1 \text { or } 0 \\
\text { indicating whether the } m \text { th relay is selected or not }\end{array}$ \\
\hline $\begin{array}{c}\text { Initialization } \\
\text { method }\end{array}$ & $\begin{array}{l}\text { Random initialization for link weights and activation } \\
\text { thresholds with range expansion coefficient } \beta_{p s}^{s e} .\end{array}$ \\
\hline $\begin{array}{c}\text { Termination } \\
\text { condition }\end{array}$ & All labeled training data sets have been exhaustively used. \\
\hline
\end{tabular}

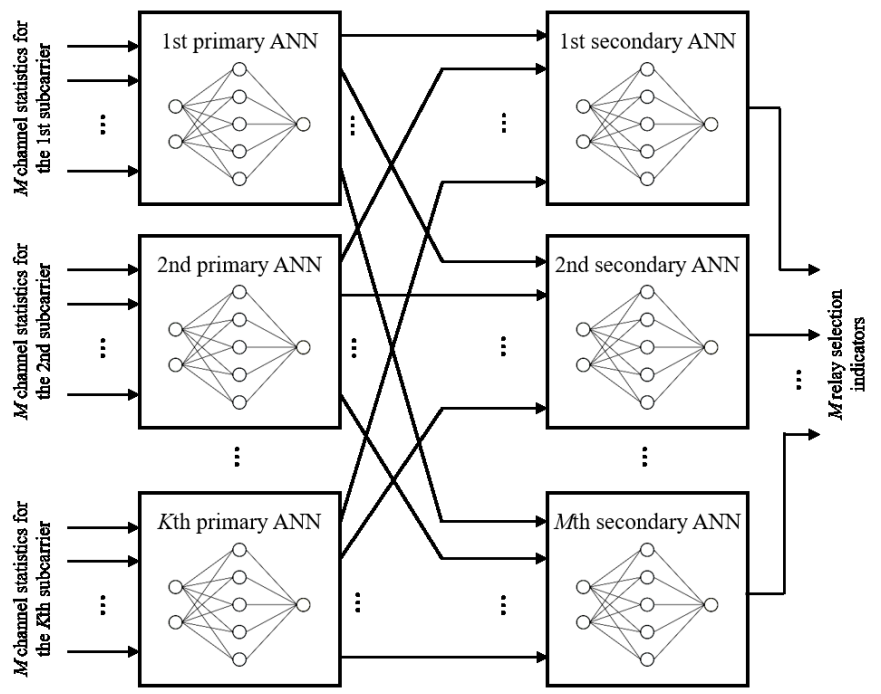

Fig. 4: CANN model for performing per-subcarrier relay selection with $K$ primary ANNs and $M$ secondary ANNs.

the classic supervised ML with detailed steps given in [22]. Therefore, the link weights and activation thresholds can be updated in an iterative way to approach the desirable set of selected relays by the per-subcarrier criterion given in (5) until all training data sets have been exhaustively utilized.

The above procedures are almost the same as those of bulk relay selection. However, the quantization function utilized for per-subcarrier relay selection is different, which can be written as $\Xi_{p s}(\cdot)$ and performs the entry-wise quantization by $H(\hat{m}-$ $1 / 2)$, where $H(\cdot)$ is the Heaviside step function. Furthermore, the outputs from the secondary ANNs in the context of persubcarrier relay selection should not be assessed individually. Rather, all $M$ outputs from $M$ secondary ANNs need to be collected and used to construct a vector $\hat{\mathbf{m}}$ whose entries of ones are associated with the selected relays and entries of zeros are associated with the other relays.

Accordingly, the PMSE and MSQE of the CANN constructed for per-subcarrier relay selection are slightly altered and defined as follows:

$$
\begin{aligned}
\mathrm{PMSE}=\mathbb{E}\{\|[\tilde{m}(1)-\hat{m}(1), \tilde{m}(2)-\hat{m}(2), & \\
& \left.\ldots, \tilde{m}(K)-\hat{m}(K)]^{T} \|_{F}^{2}\right\},
\end{aligned}
$$

and

$$
\mathrm{MSQE}=\mathbb{E}\left\{\left\|\mathbf{m}-\Xi_{p s}(\hat{\mathbf{m}})\right\|_{F}^{2}\right\},
$$

while the SMSE and RE of the CANN constructed for persubcarrier relay selection are given by the same expressions as (8) and (10) for bulk relay selection.

\section{Numerical Results and Discussion}

To verify the effectiveness and efficiency of the proposed multi-carrier relay selection enabling technique by CANN, we carry out numerical simulations and present the simulation results in this section for discussions.

\section{A. Simulation Configurations}

For simplicity, we normalize the hyperparameters by $\alpha_{b u l k}^{p r}=\alpha_{b u l k}^{s e}=\alpha_{p s}^{p r}=\alpha_{p s}^{s e}=1$ and $\beta_{b u l k}^{p r}=\beta_{b u l k}^{s e}=$ $\beta_{p s}^{p r}=\beta_{p s}^{s e}=1$. We generate $10^{7}$ training data sets by $\left\{G_{1}(m, k)\right\},\left\{G_{2}(m, k)\right\} \sim \operatorname{exprnd}(1)$ via the embedded exponentially distributed random number function on MATLAB as well as their labels by the exhaustive search according to (4) or (5). To mitigate the randomness of channel realizations and reveal the statistical nature of the proposed CANN based relay selection enabling technique, we average all simulation results over all previous trials under the identical simulation configurations for illustration.

\section{B. Bulk Relay Selection}

Based on the simulation configurations given in the above subsection, we also set the homogeneous architectures for primary and secondary ANNs and let $L_{b u l k}^{p r}=$ $4, L_{b u l k}^{s e}=2,\left[N_{b u l k}^{p r}(1), N_{b u l k}^{p r}(2), N_{b u l k}^{p r}(3), N_{b u l k}^{p r}(4),\right]^{T}=$ $[K, 10 K, 10 K, 1]^{T}$, and $\left[N_{\text {bulk }}^{\text {se }}(1), N_{\text {bulk }}^{\text {se }}(2)\right]^{T}=[M, M]^{T}$. Here, we adopt a relatively complex architecture for the primary ANN, since its functionality is complex and the precision requirement is high, i.e. its expected outputs are precise decimals between 0 and 1 . On the other hand, we adopt a simplistic architecture without even a hidden layer for the secondary ANN, because its functionality is simply classification and the expected outputs are binary.

We present the simulation results pertaining to bulk relay selection for both DF and AF cooperative relay networks with different numbers of relays and subcarriers $(M$ and $K)$ in Fig. 5. Observing the training performance evaluation metrics from Fig. 5, we can first find that all of them decrease after initial oscillation periods within the first 100 epochs by iterative training with generated training data sets with labels, which verifies the effectiveness of the proposed CANN enabling technique for bulk relay selection. Note that, at the early training stage, all four metrics could be quite small, which is caused by the lack of random realization samples at the early stage for averaging. Because the numbers of relays and subcarriers are small in our examples, it is highly possible that a randomly selected subset of relays could be close to or 
even exactly match the optimal relaying strategy, and therefore small PMSE/SMSE/MSQE/RE could sometimes appear. This does not indicate good system performance from the statistical standpoint. Second, even though all four training performance evaluation metrics converge to small values with an increasing number of training epochs, they will not approach infinitesimal in any case, because the errors are caused by the inherent nature of CANN and supervised ML and can hardly be eliminated. Meanwhile, we can also investigate the impacts of $M$ and $K$ as well as the adoption of relaying protocol on the training performance from Fig. 5. Increasing any of them results in poorer training performance, because more complicated implicit patterns between the given data sets and labels need to be discovered. On the contrary, different relaying protocols make a trivial difference on the training performance, as $\{G(m, k)\}$ is regarded as a compact entity as the input.

\section{Per-Subcarrier Relay Selection}

We initialize the CANN for per-subcarrier relay selection by the following architecture parameters: $L_{p s}^{p r}=3$, $L_{p s}^{s e}=2,\left[N_{p s}^{p r}(1), N_{p s}^{p r}(2), N_{p s}^{p r}(3)\right]^{T}=[M, 10 M, M]^{T}$, and $\left[N_{p s}^{s e}(1), N_{p s}^{s e}(2)\right]^{T}=[K, 1]^{T}$. The architecture of the primary ANN contains a single hidden layer, which is more complex than that of the secondary ANN. This is because the primary ANNs function as classifiers, while the secondary ANNs function as OR gates with imperfect inputs that are relatively simple and easy to be trained without any hidden layer [39].

We show the simulation results regarding per-subcarrier relay selection for both DF and AF cooperative relay networks with different numbers of relays and subcarriers $(M$ and $K$ ) in Fig. 6. The all-important observation from Fig. 6 is that the CANN based enabling technique also works for per-subcarrier relay selection, as all four training performance evaluation metrics keep declining with iterative training after the initial oscillation periods within the first 100 epochs. In addition, compared to the CANN for realizing bulk relay selection, the CANN of per-subcarrier relay selection is much easier to train, because all expected outputs from the primary and secondary ANNs are binary, instead of decimals. Other observations and impacts of parameters are consistent with those of the CANN of bulk relay selection.

\section{Performance Improvement Brought by the Two-Stage SF Process}

To reveal the performance improvement brought by the two-stage SF process introduced in this paper, we assume a perfectly trained CANN (i.e., MSQE $\equiv 0$ and RE $\equiv 0$ ) and test the performance of cases with different forms of input reference data. In particular, three representative cases are considered for comparison purposes:

- Case 1: Two-stage SF as stipulated Section II-A;

- Case 2: Single-stage SF in the first hop, without knowledge of the channel in the second hop;

- Case 3: No SF involved.

The average minimum channel power gains corresponding to all three cases with different system configurations are simulated and plotted in Fig. 7. From this figure, it is clear that the two-stage SF process can bring a significant performance gain for enhancing the worst subcarrier by forwarding it via the optimal relay from an end-to-end perspective. When the number of available relays increases, the performance gain goes large, which indicates that the two-stage SF process is capable of integrating more CSI sources and exploiting relays in an efficient manner. On the other hand, although there is also a performance gain brought by the single-stage SF process, the improvement is moderate and soon get saturated when increasing the number of available relays. This saturation is caused by the error propagation (for DF relaying) and improper noise amplification (for AF relaying), when the CSI of the second hop is missing. The straight lines of Case 3 without SF indicate that systems not employing SF cannot exploit the multi-source reference information to improve reliability.

\section{CONCLUSION}

To realize fast deployment and efficient exploitation of multi-carrier multi-relay selection in intelligent vehicular communication systems, we proposed an enabling technique for multi-carrier relay selection by SF and supervised ML through CANNs. We also took two classic multi-carrier relay selection schemes as examples to demonstrate how the proposed enabling technique can be implemented and benefit. Numerical results verified the effectiveness and efficiency of utilizing CANNs to perform multi-carrier relay selection. In addition, through numerical results, we obtained the insight into the statistical nature of the SF and CANN based enabling technique as well as the impacts of key system parameters on the training performance.

\section{ACKNOWLEDGMENT}

We thank the editor and the anonymous reviewers for their constructive comments, which have helped us improve the quality of the paper.

\section{REFERENCES}

[1] R. Pabst and et al, "Relay-based deployment concepts for wireless and mobile broadband radio," IEEE Communications Magazine, vol. 42, no. 9, pp. 80-89, Sept. 2004.

[2] S. Berger, M. Kuhn, A. Wittneben, T. Unger, and A. Klein, "Recent advances in amplify-and-forward two-hop relaying," IEEE Communications Magazine, vol. 47, no. 7, pp. 50-56, Jul. 2009.

[3] M. Pejanovic-Djurisic, E. Kocan, and R. Prasad, OFDM based relay systems for future wireless communications. River Publishers, 2012.

[4] J. Li, Y. Peng, Y. Yan, X. Jiang, H. Hai, and M. Zukerman, "Cognitive radio network assisted by OFDM with index modulation," IEEE Transactions on Vehicular Technology, vol. 69, no. 1, pp. 1106-1110, Jan. 2020.

[5] S. Dang, O. Amin, B. Shihada, and M.-S. Alouini, "What should 6G be?" Nature Electronics, vol. 3, no. 1, pp. 20-29, 2020.

[6] P. Guo, Y. Bai, Z. Ma, S. Wu, and S. Dang, "Relay technology for multicarrier systems: a research overview," in Proc. IEEE C3IT, Hooghly, India, Feb. 2015.

[7] L. Dai, B. Gui, and L. J. C. Jr., "Selective relaying in OFDM multihop cooperative networks," in Proc. IEEE WCNC, Kowloon, Hong Kong, China, Mar. 2007.

[8] W. Yang and Y. Cai, "Distributed relay selection in OFDM-based cooperative wireless networks," in Proc. IEEE WiCOM, Chengdu, China, Sept. 2010. 


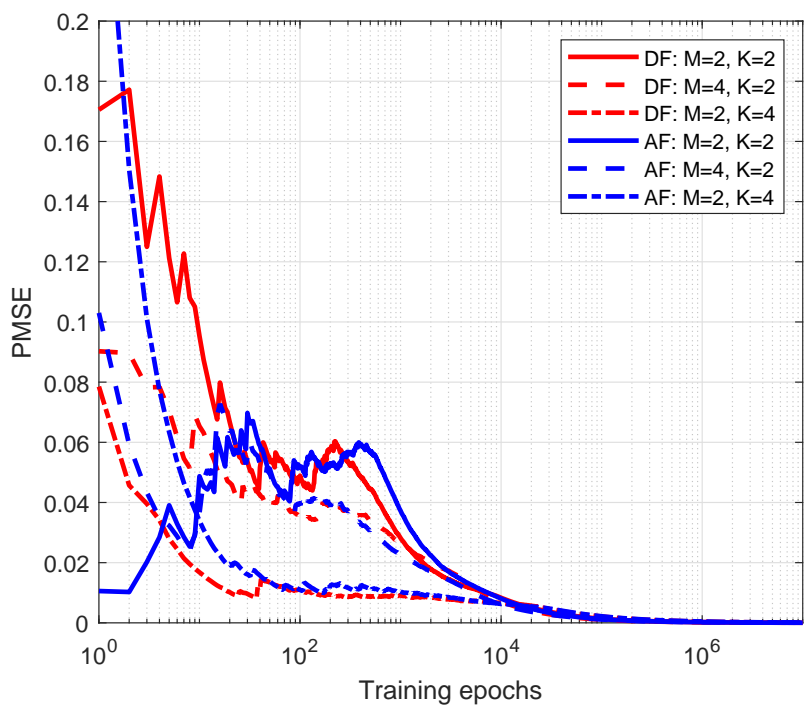

(a) PMSE

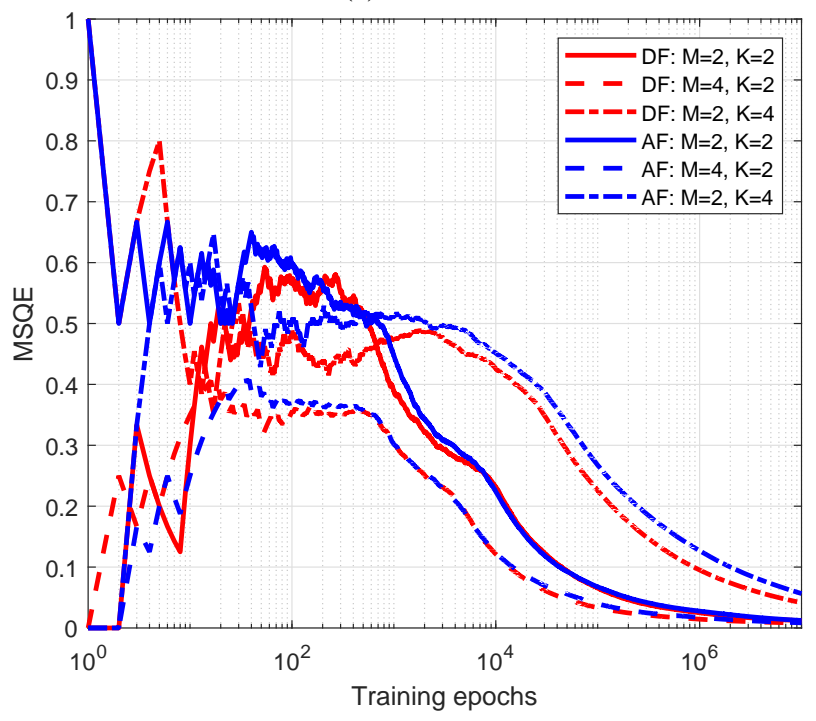

(c) MSQE

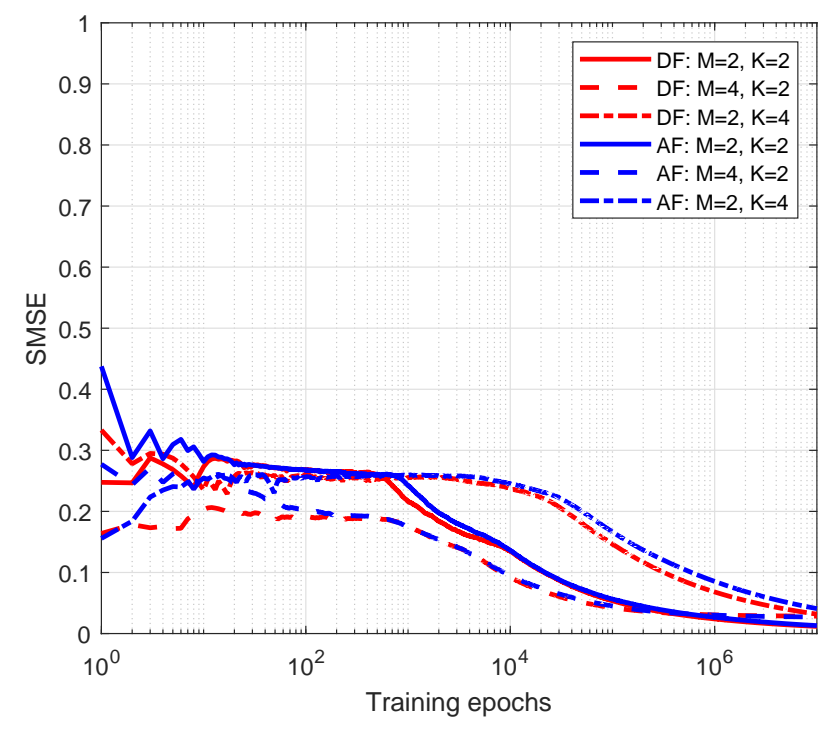

(b) SMSE

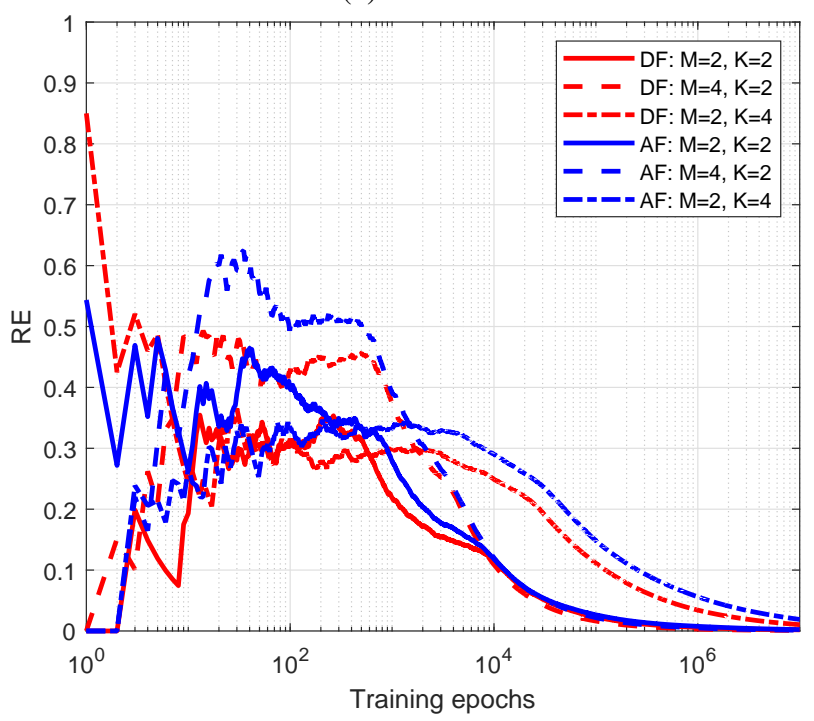

(d) RE

Fig. 5: CANN training performance indicators for bulk relay selection with different relaying protocols, numbers of relays, and numbers of subcarriers.

[9] M. Kaneko, K. Hayashi, P. Popovski, K. Ikeda, H. Sakai, and R. Prasad, "Amplify-and-forward cooperative diversity schemes for multi-carrier systems," IEEE Transactions on Wireless Communications, vol. 7, no. 5, pp. 1845-1850, May 2008.

[10] J. C. Park, T. T. Do, and Y. H. Kim, "Outage probability of OFDMbased relay networks with relay selection," in Proc. IEEE VTC Spring, Taipei, Taiwan, ROC, May 2010.

[11] Y. Ding and M. Uysal, "Amplify-and-forward cooperative OFDM with multiple-relays: performance analysis and relay selection methods," IEEE Transactions on Wireless Communications, vol. 8, no. 10, pp. 4963-4968, Oct. 2009.

[12] S. Dang, J. P. Coon, G. Chen, and D. E. Simmons, "Outage performance analysis of multicarrier relay selection for cooperative networks," in Proc. IEEE ISCC, Heraklion, Greece, Jul. 2017.

[13] S. Dang, J. P. Coon, and G. Chen, "An equivalence principle for OFDMbased combined bulk/per-subcarrier relay selection over equally spatially correlated channels," IEEE Transactions on Vehicular Technology, vol. 66, no. 1, pp. 122-133, Jan. 2017.

[14] S. Dang, D. E. Simmons, and J. P. Coon, "Comparison of multicarrier relay selection schemes in super dense networks," in Proc. IEEE CAMAD, Guildford, UK, Sept. 2015.

[15] S. Dang, G. Chen, and J. P. Coon, "Multicarrier relay selection for full-duplex relay-assisted OFDM D2D systems," IEEE Transactions on Vehicular Technology, vol. 67, no. 8, pp. 7204-7218, Aug. 2018.

[16] - "Outage performance of two-hop OFDM with index modulation and multi-carrier relay selections," IEEE Wireless Communications Letters, vol. 7, no. 6, pp. 926-929, Dec. 2018.

[17] P. Yang and F. Mu, "Outage performance of multi-carrier relay selection for full-duplex OFDM with index modulation system," IEEE Communications Letters, 2019.

[18] S. Dang, J. Li, M. Wen, and S. Mumtaz, "Distributed processing for multi-relay assisted OFDM with index modulation," IEEE Transactions on Wireless Communications, vol. 18, no. 2, pp. 1318-1331, Feb. 2019.

[19] S. Dang, J. P. Coon, and G. Chen, "Outage performance of two-hop OFDM systems with spatially random decode-and-forward relays," IEEE Access, vol. 5, pp. 27 514-27 524, 2017.

[20] Y. Li, W. Wang, and F. C. Zheng, "Combined bulk and per-tone relay selection in cooperative OFDM systems," in Proc. IEEE ICCC, Beijing, China, Aug. 2012.

[21] H. Song, J. Bai, Y. Yi, J. Wu, and L. Liu, "Artificial intelligence enabled Internet of Things: Network architecture and spectrum access," IEEE Computational Intelligence Magazine, vol. 15, no. 1, pp. 44-51, Feb. 2020.

[22] H. Dang, Y. Liang, L. Wei, C. Li, and S. Dang, "Enabling relay selection 


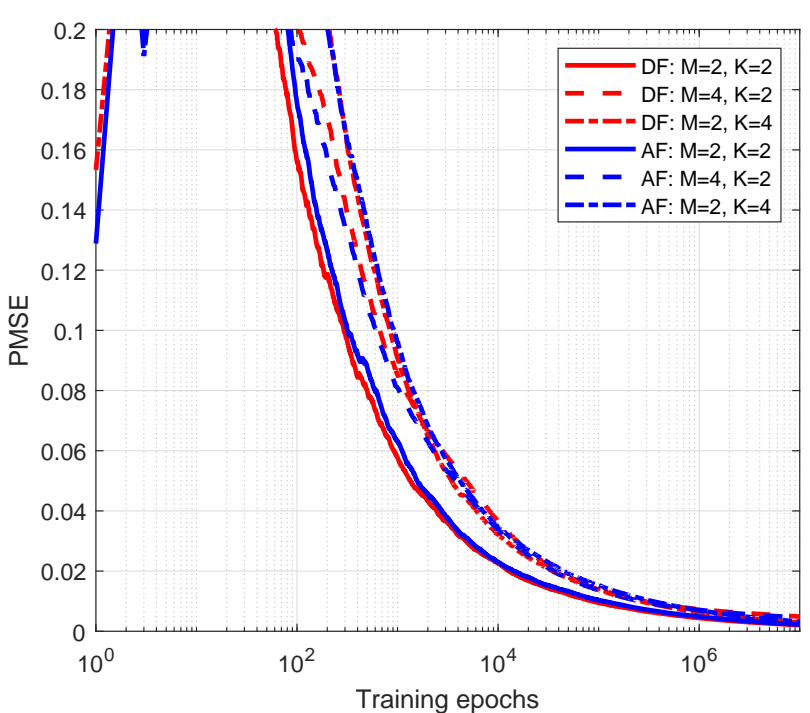

(a) PMSE

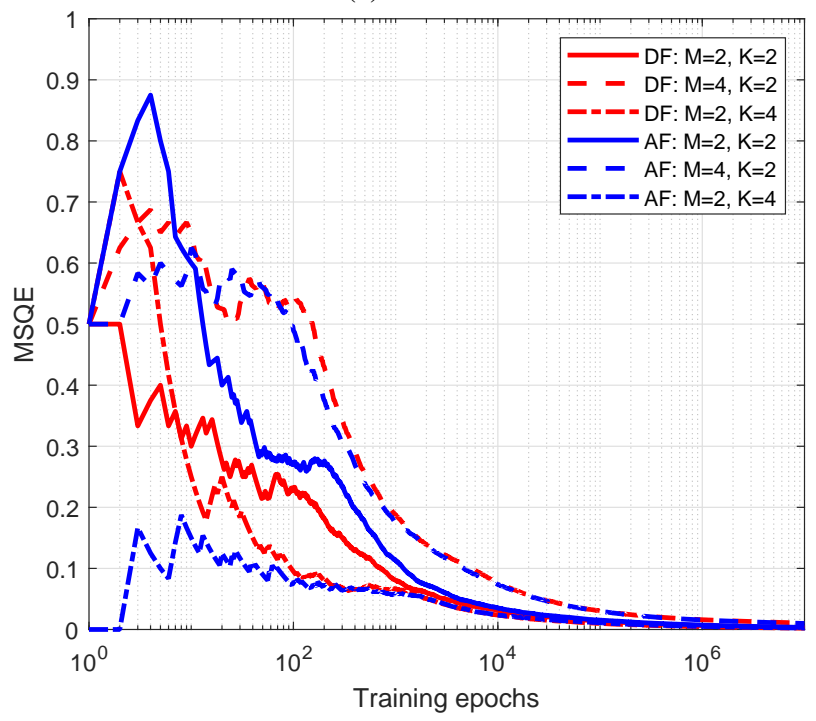

(c) MSQE

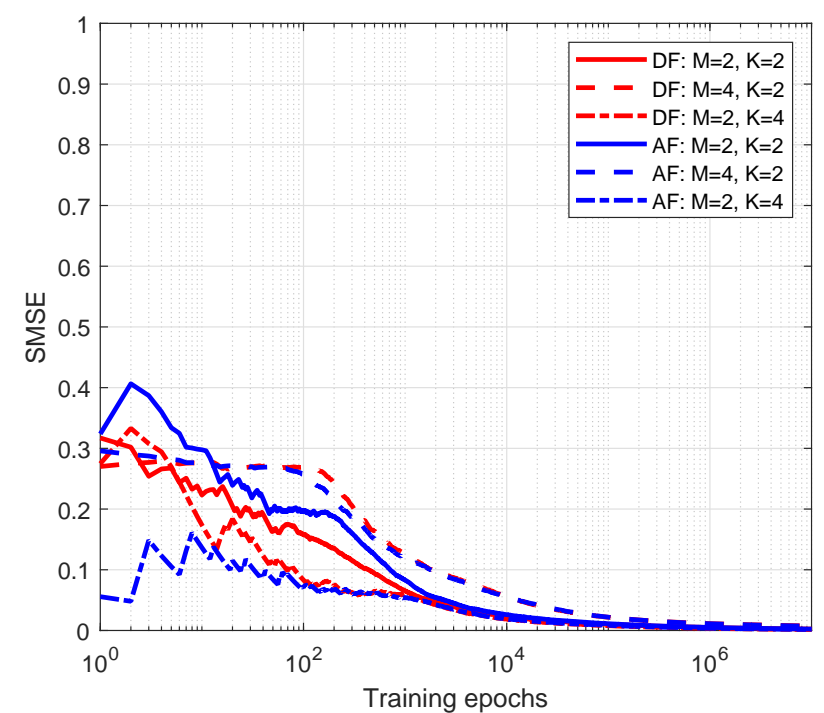

(b) SMSE

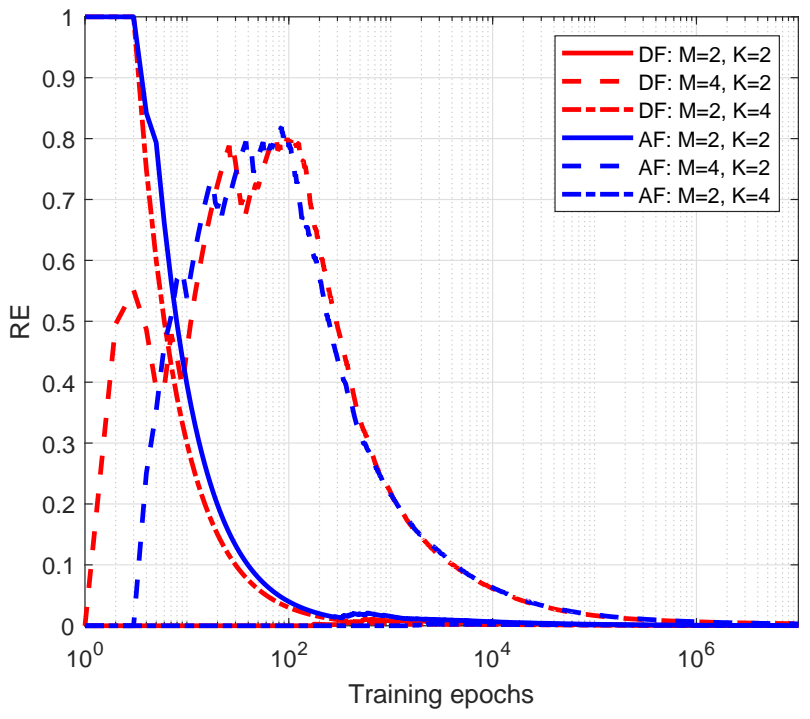

(d) RE

Fig. 6: CANN training performance indicators for per-subcarrier relay selection with different relaying protocols, numbers of relays, and numbers of subcarriers.

in cooperative networks by supervised machine learning," in Proc. IEEE IMCCC, Harbin, China, 2018, pp. 1459-1463.

[23] _ - "Artificial neural network design for enabling relay selection by supervised machine learning," in Proc. IEEE IMCCC, Harbin, China, 2018, pp. 1464-1468.

[24] Z. Wang, S. Dang, S. Shaham, Z. Zhang, and Z. Lv, "Basic research methodology in wireless communications: The first course for researchbased graduate students," IEEE Access, vol. 7, pp. 86 678-86 696, 2019.

[25] P. Papadimitratos, A. D. La Fortelle, K. Evenssen, R. Brignolo, and S. Cosenza, "Vehicular communication systems: Enabling technologies, applications, and future outlook on intelligent transportation," IEEE Communications Magazine, vol. 47, no. 11, pp. 84-95, 2009.

[26] K. Sankhe, C. Pradhan, S. Kumar, and G. Ramamurthy, "Machine learning based cooperative relay selection in virtual MIMO," arXiv preprint arXiv:1506.01910, 2015.

[27] Z. Deng, Q. Sang, Y. Gao, and C. Cai, "Optimal relay selection for wireless relay channel with external eavesdropper: A NN-based approach," in Proc. IEEE/CIC International Conference on Communications in China (ICCC), Beijing, China, Aug. 2018, pp. 515-519.

[28] T.-T. Nguyen, J.-H. Lee, M.-T. Nguyen, and Y.-H. Kim, "Machine learning-based relay selection for secure transmission in multi-hop DF relay networks," Electronics, vol. 8, no. 9, p. 949, 2019.
[29] A. Abdelreheem, O. A. Omer, H. Esmaiel, and U. S. Mohamed, "Deep learning-based relay selection in D2D millimeter wave communications," in Proc. IEEE International Conference on Computer and Information Sciences (ICCIS), Sakaka, Saudi Arabia, Apr. 2019, pp. 15 .

[30] A. Mchergui, T. Moulahi, and S. Nasri, "Relay selection based on deep learning for broadcasting in VANET," in Proc. IEEE International Wireless Communications Mobile Computing Conference (IWCMC), Tangier, Morocco, June 2019, pp. 865-870.

[31] S. Jacob, V. G. Menon, and S. Joseph, "Depth information enhancement using block matching and image pyramiding stereo vision enabled RGBD sensor," IEEE Sensors Journal, pp. 1-1, 2020.

[32] V. G. Menon and J. Prathap, "Vehicular fog computing: Challenges applications and future directions," International Journal of Vehicular Telematics and Infotainment Systems (IJVTIS), vol. 1, no. 2, pp. 15-23, 2017.

[33] M. Abbasi, M. Rafiee, M. R. Khosravi, A. Jolfaei, V. G. Menon, and J. M. Koushyar, "An efficient parallel genetic algorithm solution for vehicle routing problem in cloud implementation of the intelligent transportation systems," Journal of Cloud Computing, vol. 9, no. 1, p. 6, 2020.

[34] W. Yang and Y. Cai, "On the performance of the block-based selective 


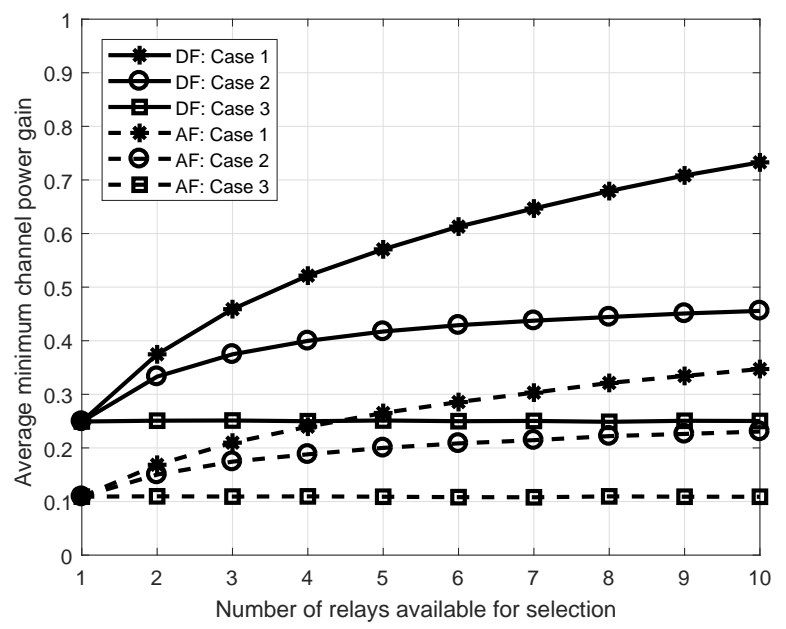

(a) Bulk relay selection

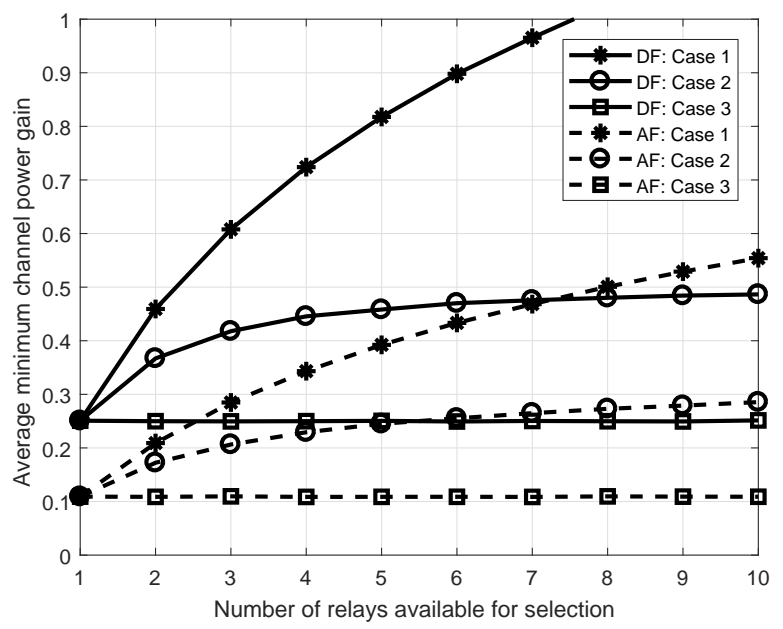

(b) Per-subcarrier relay selection

Fig. 7: Average minimum channel power gain for different forms of input reference data of perfectly trained ANN, given $K=2$.

OFDM decode-and-forward relaying scheme for $4 \mathrm{G}$ mobile communication systems," Journal of Communications and Networks, vol. 13, no. 1, pp. 56-62, Feb. 2011.

[35] D. S. Michalopoulos and G. K. Karagiannidis, "Performance analysis of single relay selection in Rayleigh fading," IEEE Transactions on Wireless Communications, vol. 7, no. 10, pp. 3718-3724, Oct. 2008.

[36] A. S. AlFuhaid, M. A. El-Sayed, and M. S. Mahmoud, "Cascaded artificial neural networks for short-term load forecasting," IEEE Transactions on Power Systems, vol. 12, no. 4, pp. 1524-1529, Nov. 1997.

[37] F. Ladstätter, E. Garrosa, B. Moreno-Jiménez, V. Ponsoda, J. M. Reales Aviles, and J. Dai, "Expanding the occupational health methodology: A concatenated artificial neural network approach to model the burnout process in Chinese nurses," Ergonomics, vol. 59, no. 2, pp. 207-221, 2016.

[38] J. Proakis and M. Salehi, Digital Communications. McGraw-Hill, 2008.

[39] M. Negnevitsky, Artificial Intelligence: A Guide to Intelligent Systems. Pearson Education Limited, 2011.

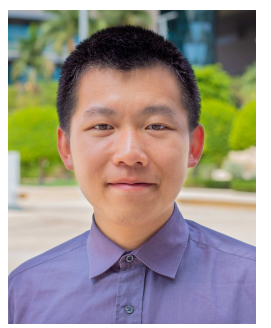

Shuping Dang (S'13-M'18) received B.Eng (Hons) in Electrical and Electronic Engineering from the University of Manchester (with first class honors) and B.Eng in Electrical Engineering and Automation from Beijing Jiaotong University in 2014 via a joint ' $2+2$ ' dual-degree program. He also received D.Phil in Engineering Science from University of Oxford in 2018. Dr. Dang joined in the R\&D Center, Huanan Communication Co., Ltd. after graduating from University of Oxford and is currently working as a Postdoctoral Fellow with the Computer, Electrical and Mathematical Science and Engineering Division, King Abdullah University of Science and Technology (KAUST). He is a co-recipient of the 'best paper' award for work presented at 2019 19th IEEE International Conference on Communication Technology. He serves as a reviewer for a number of key journals in communications and information science, including IEEE TRANSACTIONS ON WIRELESS COMMUNICATIONS, IEEE TRANSACTIONS ON COMMUNICATIONS, IEEE WIRELESS COMMUNICATIONS LetTERs, IEEE COMMUNications LetTERs, and IEEE TRANSACTIONS ON VEHICULAR TECHNOLOGY. His current research interests include novel modulation schemes, cooperative communications, and $6 \mathrm{G}$ wireless network design.

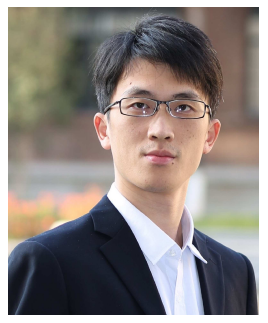

Miaowen Wen (SM'18) received the Ph.D. degree from Peking University, Beijing, China, in 2014. From 2012 to 2013, he was a Visiting Student Research Collaborator with Princeton University, Princeton, NJ, USA. He is currently an Associate Professor with South China University of Technology, Guangzhou, China, and a Hong Kong Scholar with The University of Hong Kong, Hong Kong. He has published a Springer book entitled Index Modulation for 5G Wireless Communications and more than 80 journal papers. His research interests include a variety of topics in the areas of wireless and molecular communications.

Dr. Wen was the recipient of four Best Paper Awards from the IEEE ITST'12, the IEEE ITSC'14, the IEEE ICNC'16, and the IEEE ICCT'19. He was recognized as an Exemplary Reviewer for the IEEE COMMUNICATIONS LETTERS in 2017 and the IEEE TRANSACTIONS ON COMMUNICATIONS in 2019. He has served on the Editorial Boards of the IEEE ACCESS, and the EURASIP Journal on Wireless Communications and Networking, and a Guest Editor for IEEE JOURNAL ON SELECTED AREAS IN COMMUNICATIONS (Special Issue on Spatial Modulation for Emerging Wireless Systems) and for IEEE JOURNAL OF SELECTED TOPICS IN Signal PROCESSING (Special Issue on Index Modulation for Future Wireless Networks: A Signal Processing Perspective). Currently, he is serving as an Editor for the IEEE TRANSACTIONS ON COMMUNications, the IEEE COMmUnications LetTers, and the Physical Communication (Elsevier).

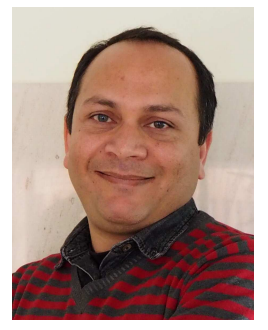

Shahid Mumtaz (SM'16) is an ACM Distinguished speaker, receipt of IEEE ComSoC Young Researcher Award (2020), IEEE Senior member, founder and EiC of IET "journal of Quantum communication", Vice-Chair: Europe/Africa Region- IEEE ComSoc: Green Communications \& Computing society and Vice-chair for IEEE standard on P1932.1: Standard for Licensed/Unlicensed Spectrum Interoperability in Wireless Mobile Networks. This standard resulted from his novel idea on "WiFi in a Licensed band". He is also a Senior 5G Consultant at Huawei, Sweden.

$\mathrm{He}$ has more than 12 years of wireless industry/academic experience. $\mathrm{He}$ has received his Master's and Ph.D. degrees in Electrical \& Electronic Engineering from Blekinge Institute of Technology, Sweden, and University of Aveiro, Portugal in 2006 and 2011, respectively. He is the author of 4 technical books, 12 book chapters, 180+ technical papers (130+ Journal/transaction, 70+ conference, 2 IEEE best paper award- in the area of mobile communications. 


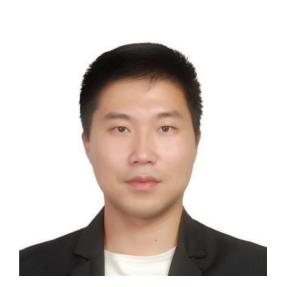

Jun Li (S'13-M'17) received the Ph.D. degrees from Chonbuk National University, Jeonju, South Korea, in 2016. He is currently an Assistant Professor with Guangzhou University, Guangzhou, China. He serves as a reviewer for IEEE TRANSACTIONS ON WIRELESS COMMUNICATIONS, IEEE TRANSACTIONS ON COMMUNICATIONS, IEEE JOURNAL OF SELECTED TOPICS IN Signal PROCESSING, IEEE JOURNAL ON SELECTED AREAS IN COMMUNICATIONS, and IEEE TRANSACTIONS ON VEHICULAR TECHNOLOGY. His research interests include spatial modulation and OFDM index modulation.

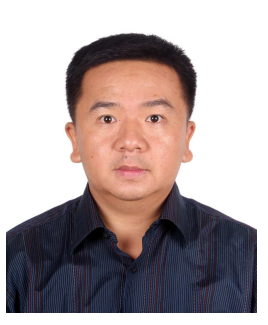

Chengzhong Li is a Qualified Senior Engineer and a Certified Expert in telecommunications by Guangxi Science \& Technology Department. He is now working as the chief engineer with the R\&D Center, Guangxi Huanan Communication Co., Ltd., Nanning, China. He has been working on the telecommunication industries for more than ten years and specializes in telecommunication network design, feasibility study, and project management. He led a number of feasibility studies of major provincial and municipal telecommunication research and construction projects. His research interests include information integration, big data, artificial intelligence, and telecommunication economics. 